\title{
Crystallization scale purification of $\alpha 7$ nicotinic acetylcholine receptor from mammalian cells using a BacMam expression system
}

\author{
Hao CHENG ${ }^{1}$, Chen FAN ${ }^{2}$, Si-wei ZHANG ${ }^{1}$, Zhong-shan WU ${ }^{1,3}$, Zhi-cheng CUI $^{2}$, Karsten MELCHER ${ }^{4}$, Cheng-hai ZHANG ${ }^{1}$, \\ Yi JIANG ${ }^{1}$, Yao CONG ${ }^{2}, \mathrm{H}$ Eric XU ${ }^{1,4, *}$ \\ ${ }^{1}$ VARI-SIMM Center, Center for Structure and Function of Drug Targets, CAS-Key Laboratory of Receptor Research, Shanghai Institute \\ of Materia Medica, Chinese Academy of Sciences, Shanghai 201203, China; ${ }^{2}$ National Center for Protein Science Shanghai, State \\ Key Laboratory of Molecular Biology, Institute of Biochemistry and Cell Biology, Shanghai Institutes for Biological Sciences, Chinese \\ Academy of Sciences, Shanghai 201210, China; ${ }^{3}$ Britton Chance Center for Biomedical Photonics, Wuhan National Laboratory for \\ Optoelectronics, Huazhong University of Science and Technology, Wuhan 430074, China; ${ }^{4}$ Laboratory of Structural Sciences, Van \\ Andel Research Institute, Grand Rapids, MI 49503, USA
}

\begin{abstract}
Aim: To report our methods for expression and purification of $\alpha 7$ nicotinic acetylcholine receptor ( $\alpha 7$-nAChR), a ligand-gated pentameric ion channel and an important drug target.

Methods: $\alpha 7$-nAChRs of 10 different species were cloned into an inducible BacMam vector with an N-terminal tag of a tandem maltosebinding protein (MBP) and a TEV cleavage site. This $\alpha 7-n A C h R$ fusion receptor was expressed in mammalian HEK293F cells and detected by Western blot. The expression was scaled up to liters. The receptor was purified using amylose resin and size-exclusion chromatography. The quality of the purified receptor was assessed using SDS-PAGE gels, thermal stability analysis, and negative stain electron microscopy (EM). The expression construct was optimized through terminal truncations and site-directed mutagenesis. Results: Expression screening revealed that $\alpha 7-n A C h R$ from Taeniopygia guttata had the highest expression levels. The fusion receptor was expressed mostly on the cell surface, and it could be efficiently purified using one-step amylose affinity chromatography. One to two milligrams of the optimized $\alpha 7$-nAChR expression construct were purified from one liter of cell culture. The purified $\alpha 7-n A C h R$ samples displayed high thermal stability with a $\mathrm{Tm}$ of $60^{\circ} \mathrm{C}$, which was further enhanced by antagonist binding but decreased in the presence of agonist. EM analysis revealed ring-like structures with a central hydrophilic hole, which was consistent with the pentameric assembly of the $\alpha 7-n A C h R$ channel.

Conclusion: We have established methods for crystallization scale expression and purification of $\alpha 7$-nAChR, which lays a foundation for high-resolution structural studies using X-ray crystallography or single particle cryo-EM analysis.
\end{abstract}

Keywords: $\alpha 7-n A C h R ;$ membrane protein; receptor expression; receptor purification; BacMam; HEK293F cells

Acta Pharmacologica Sinica (2015) 36: 1013-1023; doi: 10.1038/aps.2015.34; published online 15 June 2015

\section{Introduction}

Ligand-gated ion channels (LGICs) are among the most important therapeutic targets for various disorders. Approximately $15 \%$ of all currently available drugs act through LGICs ${ }^{[1]}$. Nicotinic acetylcholine receptors (nAChRs) comprise a major subfamily of LGICs that function at the neuromuscular junction ${ }^{[2]}$. $\mathrm{nAChRs}$ in the human nervous system have been fine-tuned by evolution to transduce a chemical signal into an electrical

\footnotetext{
* To whom correspondence should be addressed. E-mail eric.xu@vai.org

Received 2015-03-18 Accepted 2015-04-07
}

signal. nAChRs control the electrical signaling between nerve and muscle cells by opening and closing a gated membranespanning pore in response to the neurotransmitter acetylcholine $(\mathrm{ACh})$, which plays a key role in activating the receptor ion channel pore ${ }^{[3]}$.

The function of nAChRs is required to maintain appropriate neuronal activities that affect various physiological processes, including sleep, anxiety, and the processing of pain and cognitive functions ${ }^{[4]}$. nAChR activity is blocked by curare, hexamethonium, and peptide toxins, such as a-bungarotoxin, which is present in the venom of snakes and shellfish ${ }^{[5]}$. nAChRs are the molecular targets of nicotine addiction in smokers, and 
these receptors mediate the positive effects of nicotine on cognition, memory and attention in patients with schizophrenia and neurodegenerative diseases, such as Alzheimer's and Parkinson's diseases ${ }^{[6]}$.

nAChRs belong to the superfamily of pentameric ligandgated ion channels (pLGICs), known as the Cys-loop receptors, which also includes the anion-selective $\mathrm{GABA}_{\mathrm{A}} / \mathrm{GABA}_{\mathrm{C}}$ and glycine receptors and cation-selective $5-\mathrm{HT}_{3}$ serotonin receptors ${ }^{[7]}$. nAChRs are some of the most thoroughly studied members of the pLGIC family. Significant efforts in the past 20 years were devoted to structural analyses of nAChRs, which revealed that $\mathrm{nAChRs}$ are comprised of five subunits arranged around a central pore that serves as an ion channel. Seventeen $\mathrm{nAChR}$ subunits have been identified to date: a1$\alpha 10, \beta 1-\beta 4, \delta, \gamma$, and $\varepsilon^{[8]}$. Each $n A C h R$ subunit is organized into three domains: a large N-terminal extracellular ligandbinding domain (ECD) that contains the characteristic Cysloop; a transmembrane domain (TMD) consisting of four transmembrane helices (TM1-TM4) and a large cytoplasmic loop that connects TM3 and TM4; and a small C-terminal extracellular domain that follows TM $4^{[9]}$ (Figure 1). The nAChRs are broadly classified into two subtypes based on their primary sites of expression: muscle-type nicotinic receptors and neuronal-type nicotinic receptors. Muscle-type receptors are composed of $\alpha 1, \beta 1, \gamma$, and $\delta$ subunits in a 2:1:1:1 ratio or a1, $\beta 1, \delta$, and $\varepsilon$ subunits in a 2:1:1:1 ratio. The neuronaltypes are composed of heteromeric combinations of twelve different nicotinic receptor subunits, $\alpha 2-\alpha 10$ and $\beta 2-\beta 4$, for example, $(\alpha 4)_{3}(\beta 2)_{2},(\alpha 4)_{2}(\beta 2)_{3}$, or the homomeric complex $(\mathrm{a} 7)_{5}^{[10]}$.

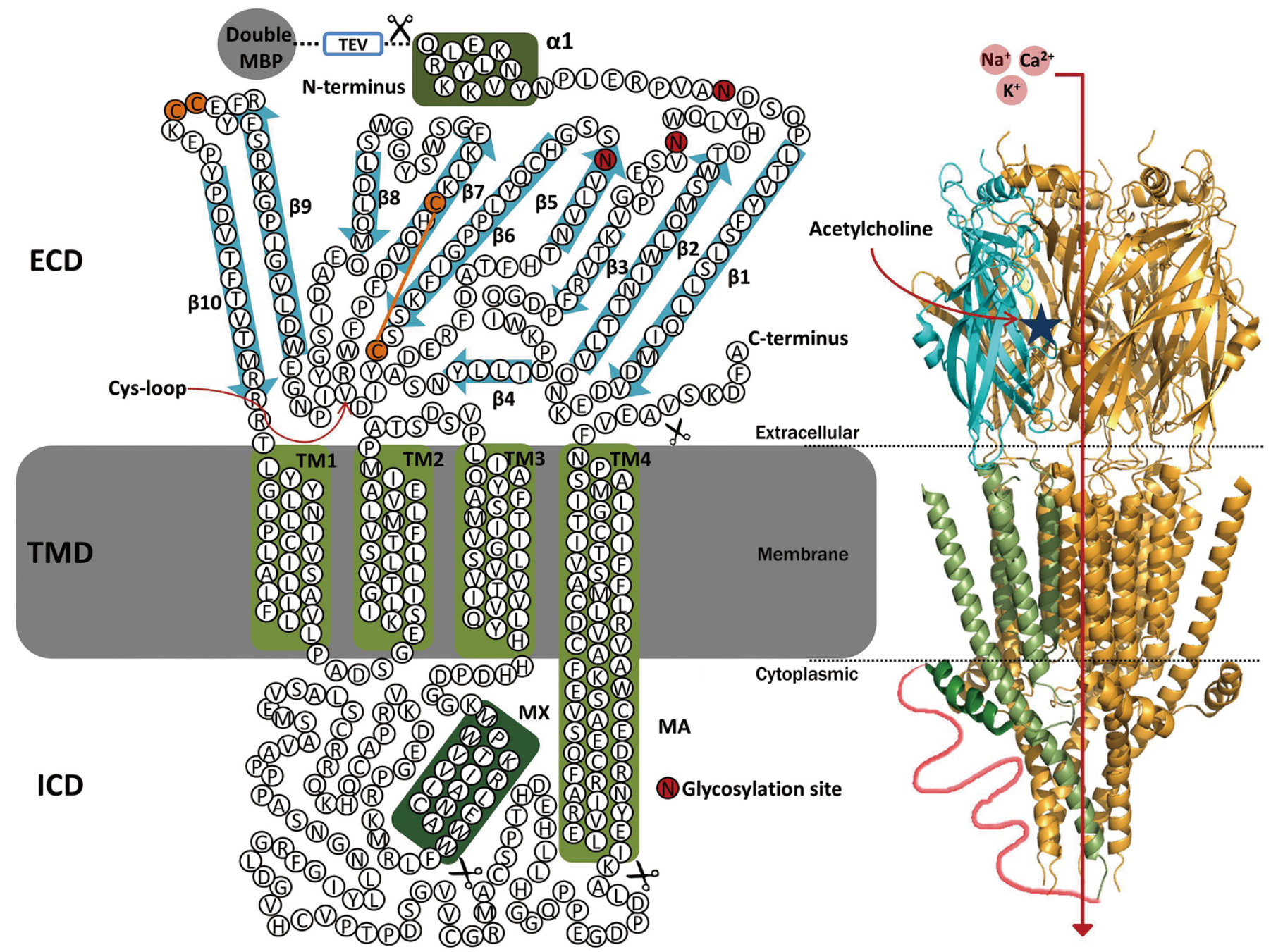

Figure 1. Schematic representation of a nAChR. The topological structure shows that a typical nAChR is composed of three distinct structural and functional entities: a large $\beta$-structured extracellular domain (ECD) that comprises the ligand-binding sites, followed by three hydrophobic transmembrane domains (TMD, TM1-TM3), a large intracellular domain (ICD), and a fourth hydrophobic transmembrane domain (TM4). N-linked glycosylation sites are highlighted in red. Schematic view of a nAChR based on the X-ray structure of the 5-HT ${ }_{3 A}$ receptor (PDB CODE: 4PIR). A dashed line schematically presents a long fragment of the cytoplasmic loop (shown only for one front subunit) that was not resolved by $\mathrm{X}$-ray structure. The double-MBP tag and TEV protease cleavage site used in this work are schematically represented. 
Most of the structural information on nAChRs was obtained for ECD, which contains the orthosteric ligand-binding site. The pentameric structures of acetylcholine-binding protein (AChBP) provide crucial insight into ligand recognition and the ligand-induced conformational changes that trigger channel opening ${ }^{[11]}$. The X-ray structure of a chimeric ECD of the human a7-nAChR with AChBP provides an invaluable atomic model for a7 ECD assembly ${ }^{[12]}$. Our knowledge of the overall topology of the TMD and intracellular domain (ICD) of nAChRs have largely relied on a $4 \AA$ cryo-EM model of the Torpedo marmarota $\mathrm{nAChR}^{[13]}$, until recent high-resolution structures of the homologous bacterial pLGICs from Erwinia chrysanthemi (ELIC) $)^{[14]}$ and Gloebacterviolaceous (GLIC) ${ }^{[15]}$ were obtained. The Caenorhabditis elegans glutamate-gated chloride channel $(\mathrm{GluCl})^{[16]}$ was the first eukaryotic pLGICs structure solved, and it provided crucial insights into channel gating and ion permeation. Two groups presented the X-ray structures of two mammalian Cys-loop receptors in 2014: the human $\mathrm{GABA}_{\mathrm{A}}$ receptor ${ }^{[17]}$ and the mouse serotonin $5-\mathrm{HT}_{3}$ receptor $^{[18]}$. These two structures offer the newest insights into the assembly and signaling mechanisms of pLGICs and enhance our current understanding of neurotransmission.

The endogenous ligand acetylcholine is a pan agonist that activates all nAChR subtypes. Similarly, many nAChR ligands, such as nicotine and epibatidine, target more than one nAChR subtype ${ }^{[19]}$. The lack of specificity of some nicotinic agonists is well known, and it is a potential problem for the treatment of illnesses that require the targeting of a specific nAChR subtype ${ }^{[20]}$. The identification of a more subtypeselective $\mathrm{nAChR}$ drug that is devoid of side effects is one goal of drug discovery research, and the structure of the fulllength $\mathrm{nAChR}$ would provide critical insight for the rational design of specific $n A C h R$ ligands ${ }^{[21]}$. However, structural studies have been hampered by difficulties in obtaining sufficient quantities of highly purified $\mathrm{nAChRs}$ for crystallization screening. We report our extensive efforts in the expression and purification of nAChRs. We obtained milligram amounts of purified $\mathrm{nAChR}$ protein for crystallization and structural determination using a BacMam expression system in HEK293F cells.

\section{Materials and methods} Construct design and molecular cloning

The original amino acid sequences of a7-nAChRs were aligned using the ClustalW program ${ }^{[22]}$. The coding regions of a7-nAChRs from 10 different species were synthesized based on sequence alignment. We designed a series of modified constructs according to the alignment and published structure information. All of the original and modified a7-nAChR cDNAs were cloned into EcoRI/NotI-digested BacMam mammalian cell expression vectors (BacMam expression system, Invitrogen, Cergy Pontoise, France) using conventional molecular cloning technology. An N-terminal double-MBP tag and a TEV cleavage sequence were introduced into the vector to facilitate the expression and purification of the target membrane proteins. The sequences of all clones were confirmed using automated DNA sequencing.

\section{Cell culture and transfection}

For routine cell culture, HEK293F cells (FreeStyle293F cells, Invitrogen) were grown as an adherent monolayer in $175 \mathrm{~cm}^{2}$ tissue culture flasks containing $10 \mathrm{~mL}$ of high glucose Dulbecco's modified Eagle's medium (DMEM) supplemented with $10 \%(v / v)$ fetal bovine serum (Gibco, Invitrogen). The cells were cultured at $37^{\circ} \mathrm{C}$ and $95 \%$ air $/ 5 \% \mathrm{CO}_{2}$ in a humidified incubator. HEK293F cells were transfected using a modified insect cell virus (baculovirus) in accordance with the commercial kit's instruction, and recombinant baculoviruses were obtained following the manufacturer's protocol (BacMam expression system, Invitrogen).

\section{Small-scale expression and construct screening}

The HEK293F cells were resuspended and spread onto a 12-well cell culture plate for small-scale expression. The BacMam viruses were transfected into cells at a 1:10 $(v / v)$ ratio. The cells were induced with $1 \mu \mathrm{g} / \mathrm{mL}$ doxycycline (SigmaAldrich, St Louis, MO, USA) $2 \mathrm{~h}$ later and harvested 18-24 h following induction. The transfected cells were solubilized, and the expression levels of the target protein were detected by Western blot and an anti-MBP monoclonal antibody (Prospec, East Brunswick, NJ, USA). Bacmids with high protein yields were selected for large-scale expression.

\section{Large-scale expression and purification of recombinant $\alpha 7$ - nAChRs}

\section{Scale-up and harvesting}

For large-scale expression, HEK293F cells from $175 \mathrm{~cm}^{2}$ culture flasks were trypsinized and transferred to a $2.8 \mathrm{~L}$ shake flask containing $1 \mathrm{~L}$ of the serum-free suspension medium CDM4HEK293 (Hyclone, Logan, UT, USA) plus $4 \mathrm{mmol} / \mathrm{L}$ Glutamax-1/20 mmol/L HEPES. Typically, $2.8 \mathrm{~L}$ shake flask cultures were seeded at $1 \times 10^{6}$ cells $/ \mathrm{mL}$ and transduced by recombinant baculoviruses when the cell density reached $4 \times 10^{6}$ cells $/ \mathrm{mL}$. pLGIC expression was induced with $1 \mu \mathrm{g} / \mathrm{mL}$ doxycycline. The cells were collected $24 \mathrm{~h}$ after transduction and pelleted from the medium using centrifugation at $2000 \times g$ for $20 \mathrm{~min}$. The cell pellets were stored at $-80^{\circ} \mathrm{C}$ until used.

\section{Solubilization of membrane proteins}

Approximately $1 \mathrm{~L}$ of the frozen cell pellets were resuspended in $50 \mathrm{~mL}$ of lysis buffer, which consisted of $10 \mathrm{mmol} / \mathrm{L}$ Tris/ $\mathrm{HCl}$ (pH 7.4), $10 \mathrm{mmol} / \mathrm{L} \mathrm{KCl}, 10 \mathrm{mmol} / \mathrm{L} \mathrm{MgCl}_{2}$ with 5 $\mathrm{mmol} / \mathrm{L}$ iodoacetamide and a protease inhibitor cocktail (Roche), and incubated in ice for $20 \mathrm{~min}$. The cells were homogenized for 5 min using a dounce homogenizer with a tightly fitting pestle (Thermo Scientific, Rockford, IL, USA). The lysate was centrifuged at $100000 \times g$ for $40 \mathrm{~min}$, and the pellets were resuspended in $20 \mathrm{~mL}$ solubilization buffer consisting of $20 \mathrm{mmol} / \mathrm{L}$ Tris/ $\mathrm{HCl}$ (pH 7.4), $150 \mathrm{mmol} / \mathrm{L} \mathrm{NaCl}$, $1 \%$ n-decyl- $\beta$-D-maltopyranoside (DDM, Affymetrix, Santa Clara, CA, USA) plus a protease cocktail (Roche, Basel, Switzerland), followed by gentle agitation for $2 \mathrm{~h}$ to solubilize the 
membranes. Insoluble material was removed using centrifugation for $40 \mathrm{~min}$ at $100000 \times \mathrm{g}$ followed by filtration through a $0.45-\mu \mathrm{m}$ filter.

\section{Affinity purification and size-exclusion chromatography of $\alpha 7$ - nAChRs}

Solubilized a7-nAChRs were purified using affinity chromatography and a $5 \mathrm{~mL}$ amylose column (GE Healthcare). The amylose resin was equilibrated with 5 column volumes of

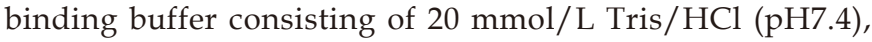
$150 \mathrm{mmol} / \mathrm{L} \mathrm{NaCl}, 0.05 \%$ DDM before loading. Solubilized a7-nAChRs were loaded onto the column and bound overnight using continuous rotation. The amylose resin was washed with 20 column volumes of binding buffer and eluted with 3 column volumes of the same buffer supplemented with $10 \mathrm{mmol} / \mathrm{L}$ maltose. Concentrations of the elution fractions containing the purified a7-nAChR were determined using a Bradford assay and an appropriate standard curve. The fusion nAChR protein was cut using a TEV Protease (Sigma) at a ratio of 1:500 $(w / w)$ for $4 \mathrm{~h}$. The protein was concentrated to approximately $5 \mathrm{mg} / \mathrm{mL}$ and further purified using a Superose 6 10/300 GL gel filtration column (GE Healthcare) equilibrated in binding buffer. Protein fractions were identified at $O D_{280}$, and a7-nAChR fractions with the affinity tag removed were pooled and concentrated for subsequent analysis.

\section{Characterization of the $\alpha 7-\mathrm{nAChR}$}

\section{Fluorescence microscopy}

An N-terminal superfolder GFP tag was introduced into the BacMam a7-nAChR vector. HEK293F cells were grown in routine cell culture, and the tagged receptor was expressed by the addition of a BacMam virus at a 1:10 $(v / v)$ ratio and 1 $\mu \mathrm{g} / \mathrm{mL}$ doxycycline (Sigma) 18-24 h before measurement. GFP fluorescence images were recorded using a customized, wide-field epifluorescence microscope (Nikon TE2000, Nikon, Canada) with appropriate excitation and emission filters.

\section{SDS gel electrophoresis}

Purified a7-nAChRs were analyzed using sodium dodecyl sulfate-polyacrylamide gel electrophoresis (SDS-PAGE) in a 5\% polyacrylamide stacking gel and $10 \%$ polyacrylamide separation gel. The proteins in gels were visualized by staining with Coomassie brilliant blue dye.

\section{Western blotting}

Western blotting was conducted as described in the kit's manual (Bio-Rad). The transfected cells from a 12-well cell culture plate were harvested and lysed with lysis buffer $(50 \mu \mathrm{L} /$ well) containing $20 \mathrm{mmol} / \mathrm{L}$ Tris- $\mathrm{HCl}(\mathrm{pH} 7.4), 150 \mathrm{mmol} / \mathrm{L}$ $\mathrm{NaCl}, 1 \% \mathrm{DDM}$ plus protease cocktail (Roche). Samples (20 $\mu \mathrm{L})$ containing total protein were separated using SDS-PAGE and transferred to PVDF membranes, followed by blocking and incubation with an anti-MBP primary antibody (1:1000) at $4^{\circ} \mathrm{C}$ overnight. The blots were incubated with a horseradish peroxidase-conjugated secondary antibody (1:2000) at room temperature for $1 \mathrm{~h}$ and developed using a Bio-Rad system.

\section{Thermal stability assay}

Thermal stability assays were performed as previously described $^{[23]}$. N-[4-(7-diethylamino-4-methyl-3-coumarinyl) phenyl]maleimide (CPM) dye (Invitrogen) was dissolved in DMSO (Sigma) at $4 \mathrm{mg} / \mathrm{mL}$ as a stock solution, which was kept at $-80^{\circ} \mathrm{C}$ and diluted 1:40 in a dye dilution solution [10 $\mathrm{mmol} / \mathrm{L}$ buffer, $150 \mathrm{mmol} / \mathrm{L} \mathrm{NaCl}, 0.05 \%$ ( $w / v)$ DDM] before use. The thermal denaturation assay was performed in a total volume of $200 \mu \mathrm{L}$ in a quartz fluorometer cuvette (Starna Cells, Inc, Atascadero, CA, USA). The purified receptor $(4 \mu \mathrm{g})$ was diluted in the appropriate buffer and ligand solution to a final volume of $200 \mu \mathrm{L}$. The concentration of ligand used was tenfold the $K_{\mathrm{i}}$ value. The diluted dye solution $(5 \mu \mathrm{L})$ was added to the protein solution and incubated for $30 \mathrm{~min}$ at $4^{\circ} \mathrm{C}$ in the dark. The mixed solution was transferred to a cuvette, and the data were collected using a Cary Eclipse spectrofluorometer (Varian, USA) with a temperature ramping rate of $1^{\circ} \mathrm{C} / \mathrm{min}$ over a temperature range from $20^{\circ} \mathrm{C}$ to $90^{\circ} \mathrm{C}$. The excitation wavelength was $387 \mathrm{~nm}$, and the emission wavelength was $463 \mathrm{~nm}$. The stability data were processed, and melting temperatures $(\mathrm{Tm})$ were obtained using Prism (GraphPad Software, La Jolla, CA, USA).

\section{Electron microscopy}

A purified a7-nAChR $(0.3 \mathrm{mg} / \mathrm{mL})$ sample $(5 \mu \mathrm{L})$ was deposited onto a glow-discharged 400 mesh continuous carbon grid (Beijing Zhongjingkeyi Technology, Beijing, China). The sample was stained with $2 \%$ uranyl formate and air-dried. The data were recorded on a Tecnai G2 F20 TWIN transmission electron microscope (FEI) equipped with a field-emission gun operated at $200 \mathrm{kV}$. Images were recorded at $71000 \times$ microscope magnification on a $4 \mathrm{k} \times 4 \mathrm{k}$ Eagle CCD camera with a pixel size of $1.15 \AA$ per pixel. The defocus ranged from -1.0 to $-1.5 \mu \mathrm{m}$. A total of 1071 particles were boxed out from 81 micrographs using the e2boxer.py program in EMAN2.1 $1^{[24]}$. Reference-free 2D image classification was performed using the EMAN1.9 program refine2d.py ${ }^{[25]}$.

\section{Results}

An efficient mammalian expression system for the human $\alpha 7$ nAChR

We previously tried various bacterial and baculovirus-insect cell expression systems, but all of these systems failed to express human a7-nAChR. Therefore, we turned our attention to mammalian expression systems. The use of Human Embryonic Kidney 293 (HEK293) cells to express full-length human membrane proteins has become increasingly popular because these cells provide a near-native protein folding and lipid environment ${ }^{[26]}$. Several mammalian ion channels, including transient receptor potential channel (TRP) ${ }^{[27]}$ and the homopentameric $5-\mathrm{HT}_{3}$ receptor, have been expressed in HEK293 cells, which suggested a potential solution to the poor expression of a7-nAChR ${ }^{[28]}$.

We used the BacMam expression system from Invitrogen for mammalian expression because it offers high levels of protein expression $^{[29]}$. A codon-optimized cDNA of full-length human 
a7-nAChR was cloned into the mammalian cell BacMam expression vector, and the baculovirus was generated from insect cells using standard methods (Figure 2A). We introduced the coding region for an N-terminal superfolder GFP tag into the BacMam vector to quickly assess the efficiency of this system (Figure 2B). The adherent monolayer HEK293F cells displayed a uniform and robust expression of a7-nAChR one day after Bacmid transfection, which was detected as high levels of GFP fluorescence signal on the surface of HEK293F cells (Figure 2C).

\section{Design and optimization of the $\alpha 7-n A C h R$ expression construct}

Once the initial expression system for $\alpha 7-n A C h R$ was established, it was important to screen various expression constructs for expression levels and protein behavior to assess whether these expression constructs were suitable for structural studies. Several variables exert a pronounced effect on expression levels, homogeneity, and the crystallizability of membrane proteins, including affinity tag type and location, codon use, detergent types, truncations, paralogues or orthologues, ligands, and lipids ${ }^{[26]}$. Fortunately, many of these variables can be initially assessed using proteins that are expressed in transiently transfected HEK293F cells.

We first screened the expression levels of a7-nAChR from 10 different species selected by phylogenetic analyses of amino acid sequences (Figure 3 ). The synthetic cDNA constructs encoding the full-length a7-nAChRs were codon-optimized for expression in mammalian cells and cloned into BacMam vectors. We introduced an N-terminal double MBP-tag and a TEV cleavage sequence, instead of an sfGFP-tag, into the vector for membrane protein expression and purification (Figure $4 \mathrm{~A})$. The a7-nAChR fusion proteins were expressed in a small-scale in HEK293F cells, and the expression levels were efficiently detected using Western blotting. Among the 10 different species tested, a7-nAChRs from the zebra finch (Taeniopygia guttata) were expressed at the highest levels (Figure 4B).

We screened the expression of various constructs with $\mathrm{N}$-terminal and C-terminal truncations, removal of $\mathrm{N}$-linked glycosylation sites, deletion of the predicted flexible intracellular loop between TM3 and TM4, and introduction of chimeric domains to further optimize the expression constructs (Figure 4). We designed 5 different lengths of the N-terminal truncation and 8 different lengths of the C-terminal truncation based on sequence alignment. The data showed that any truncation at the N-terminus reduced protein expression (Figure 4C), and most C-terminal truncations increased expression. The construct that included a deletion of 6 amino acids from the C-terminus exhibited the best expression (Figure 4D). The C-terminal 6 amino acids are not conserved among species, and these amino acids are completely missing in some species (Figure 3).

The a7-nAChR contains 3 putative N-linked glycosylation sites, and the receptor seemed to be heavily glycosylated when expressed in HEK293F cells, as indicated from Western blot (Figure 4E) and SDS-PAGE (Figure 5) analyses. Heterogeneity of the glycosylation pattern in the HEK293F-derived protein

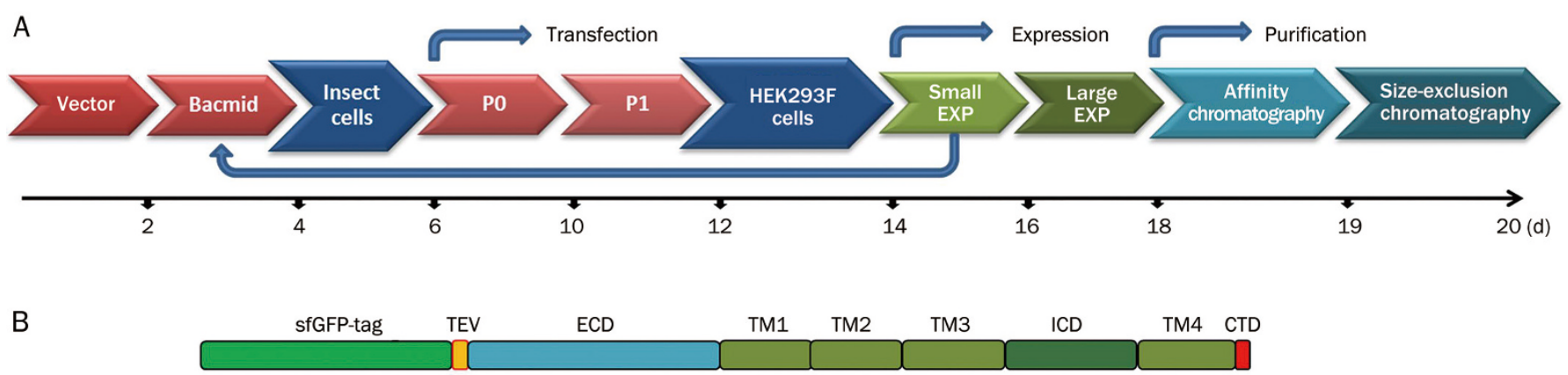

C

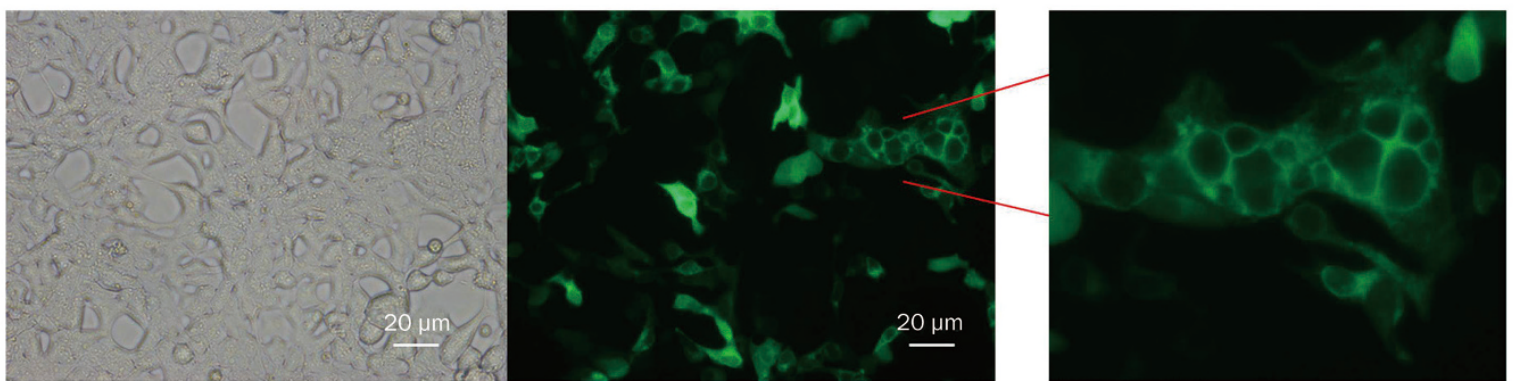

Figure 2. Recombinant expression of the human $\alpha 7-n A C h R$ in HEK293F cells. (A) A flow chart of $\alpha 7-n A C h R$ expression and purification using the BacMam system. (B) Schematic depiction of the N-terminally GFP-tagged expression construct, in which a superfolder GFP (sfGFP)-tag was introduced into the BacMam vector. (C) The surface expression of the superfolder GFP-tagged $\alpha 7$-nAChR in HEK293F cells was demonstrated using fluorescence microscopy. 


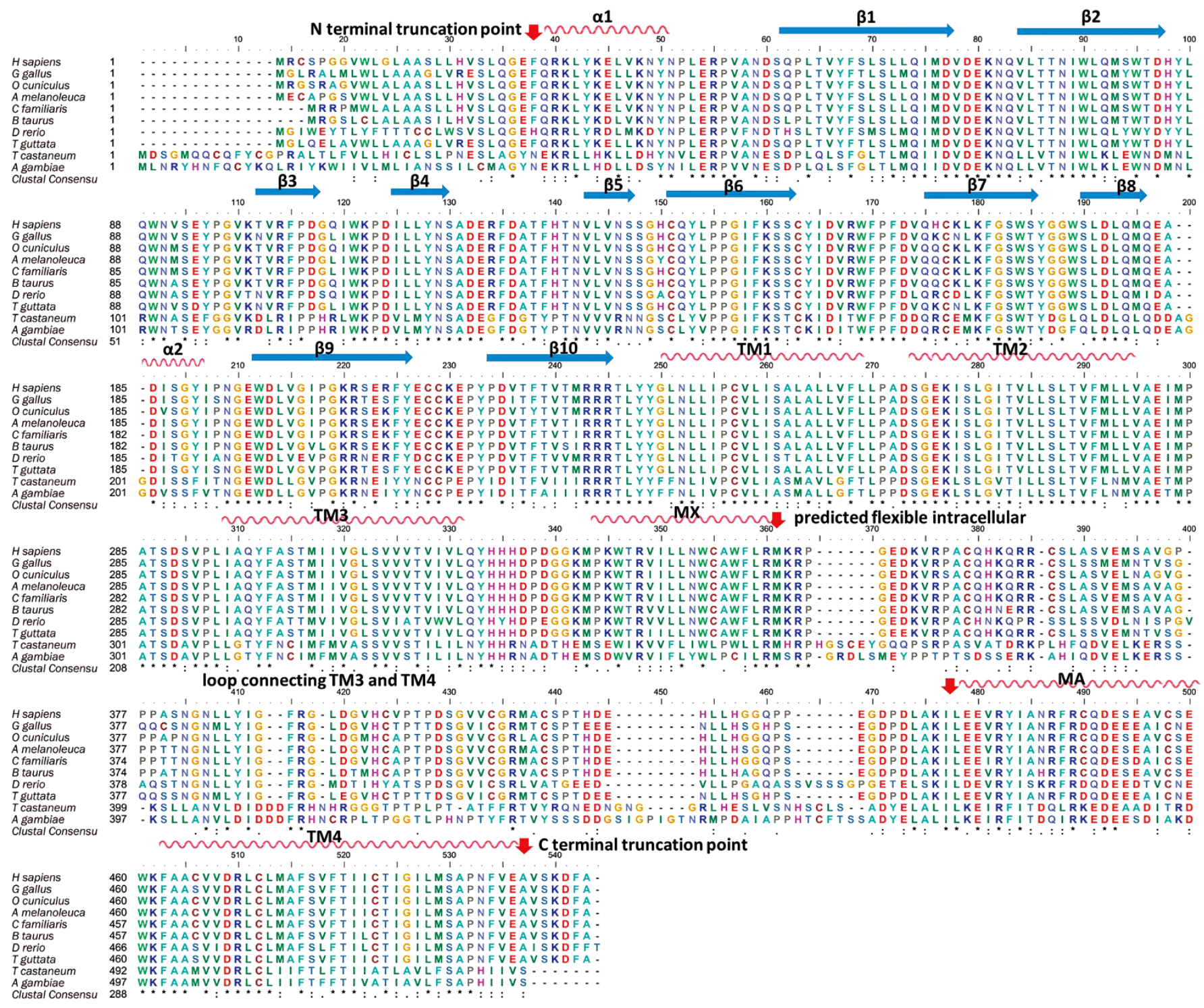

Figure 3. Sequence alignment of $\alpha 7$ nAChRs from 10 different species. Secondary structures are shown schematically above the sequences, based on the X-ray structure of the $5-\mathrm{HT}_{3 \mathrm{~A}}$ receptor (PDB CODE: 4PIR). Some of the residues important for construct optimization are highlighted by red arrows. NCBI Reference Sequence: H sapiens, NP_000737.1; G gallus, NP_989512.2; O cuniculus, XP_002718333.1; A melanoleuca, XP_002929231.1; C familiaris, XP_545813.2; B taurus, NP_776940.1; D rerio, NP_957513.1; $T$ guttata, XP_002196642.1; $T$ castaneum, ABS86911.1; A gambiae, XP_314691.2.

may compromise crystallization. Therefore, we mutated the $\mathrm{N}$-glycosylation sites (3 per subunit, total of 15 in each homopentamer) to remove the heterogeneity of the carbohydrates. These mutated receptors (N46A, N90A, and N133A) displayed only slightly reduced expression levels with a reduced molecular weight in SDS gels, possibly due to the absence of glycosylation (Figure 4E).

The $\mathrm{a} 7-\mathrm{nAChR}$ is predicted to have a large flexible intracellular loop of 100-150 amino acids connecting TM3 and TM4 (Figure 2). This intracellular loop is not as conserved as other regions of the receptors, and deletion of the predicted flexible intracellular loop between TM3 and TM4 increases protein expression but does not affect protein function ${ }^{[30]}$. Therefore, we introduced a linker region with 4 to 7 copies of the tripeptide Gly-Ser-Ala (GSA) to replace the intracellular loop of TM3 and TM4. The data showed that all of these GSA-linked receptors had increased expression (Figure 4F).

\section{Large-scale expression and affinity purification}

We used suspension cell culture for large-scale expression because it allowed mass production of the receptor. The HEK293F cell line expressing the receptor displayed a high growth rate after adaptation to the suspension culture in small volumes, and it reached $2 \times 10^{6}$ cells $/ \mathrm{mL}$ with virtually no cell death. One-liter suspension cultures for large-scale expression were induced at $4 \times 10^{6}$ cells $/ \mathrm{mL}$, and the cells were harvested 
A

Double-MBP tag TEV ECD

B

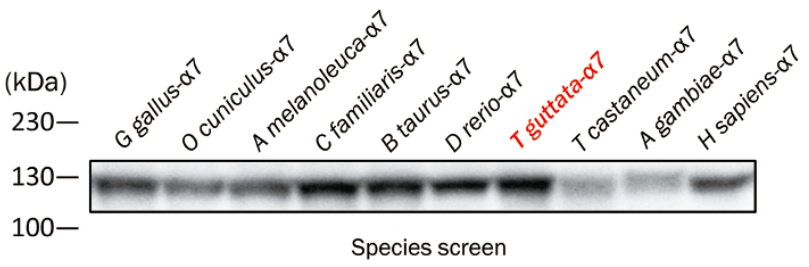

C

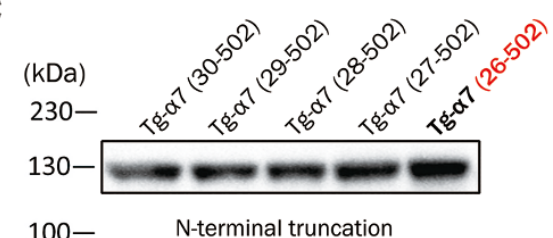

E

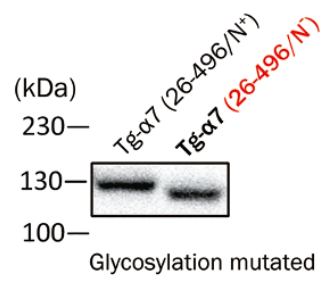

D

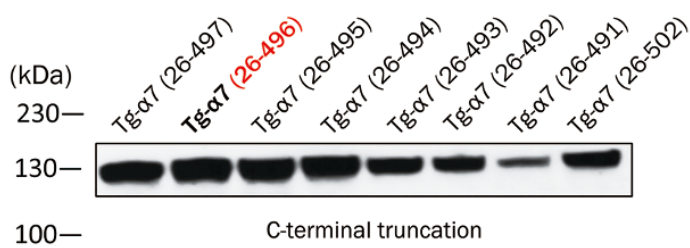

F

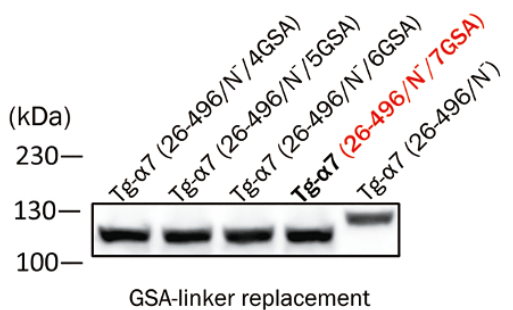

Figure 4. Design and optimization of the $\alpha 7$-nAChR expression construct. (A) Schematic depiction of the N-terminal double-MBP expression construct. (B-F) Western blot analysis of $\alpha 7$ nAChRs from 10 different species (B), N-terminally truncated $\alpha 7$ nAChR (C), C-terminally truncated $\alpha 7$ nAChR (D), glycosylation of wild-type $\left(\mathrm{N}^{+}\right)$and mutated $\left(\mathrm{N}^{-}\right)$receptor $(\mathrm{E})$, and the receptor with a GSA-linker replacement of the intracellular loop (F).

one day later. This technique typically yielded $10 \mathrm{~g}$ of biomass and $2 \mathrm{~g}$ of isolated membranes that contained up to $6 \mathrm{mg}$ of a7-nAChR fusion protein. We routinely generated $1-2 \mathrm{mg}$ of pure un-tagged $\mathrm{a} 7$-nAChR protein using the purification protocol described below.

The detergent DDM is well suited for the extraction of large amounts of the a7-nAChR fusion protein from membranes, and it was used at a concentration of $1 \%(w / v)$ for membrane solubilization and $0.05 \%$ for subsequent purification steps. The N-terminal double-MBP tag allowed purification of the receptor fusion protein from the membrane extract in a single step with an acceptable degree of purity (Figure 5B). In addition to serving as an affinity tag, the double-MBP tag was also designed to increase protein solubility and improve the yield of purification. Using this construct in a BacMam system, we obtained the pure fusion receptor with a typical yield of $6 \mathrm{mg}$ from one liter of virus-transfected cells, and the MBP tag of the fusion receptor was removed by digestion with the TEV protease (Figure 5B).

We performed size-exclusion chromatography at the last stage of the a7-nAChR purification using a Superose $610 / 300$ column, which is capable of separating proteins with sizes up to ten million Daltons, to isolate the pentameric nAChR from non-native aggregated species and the double-MBP tag. We observed a clear peak that corresponded to the fusion pentameric $\alpha 7-n A C h R$ receptor in the elution fraction from the affinity purification step (Figure 5A). TEV cleavage of the tag changed the elution profile that yielded a peak at approximately 13 $\mathrm{mL}$. This peak corresponds to an apparent molecular mass of approximate $290 \mathrm{kDa}$ based on a calibration of the column (Figure 5A), which corresponded to a pentameric complex of $\mathrm{nAChR}$ that is composed of a monomeric subunit with a predicted molecular weight of $58 \mathrm{kDa}$. The protein peak seemed quite broad (Figure 5), whereas the glycosylation site-mutated receptor appeared to have a sharper peak (Figure 6). The purified nAChR migrated with a mobility that between the 50 and $70 \mathrm{kDa}$ protein markers in SDS-PAGE gels (Figures 5, 6).

Thermal stability and electron microscopy analysis of the purified receptor

High stability is an important parameter for the crystallization of membrane protein complexes. We used a fluorescencebased thermal stability assay with CPM dye, which dramatically increases its fluorescence intensity upon binding to accessible cysteines, to determine the stability of the purified receptor. Buried cysteine residues were inaccessible to CPM 
A

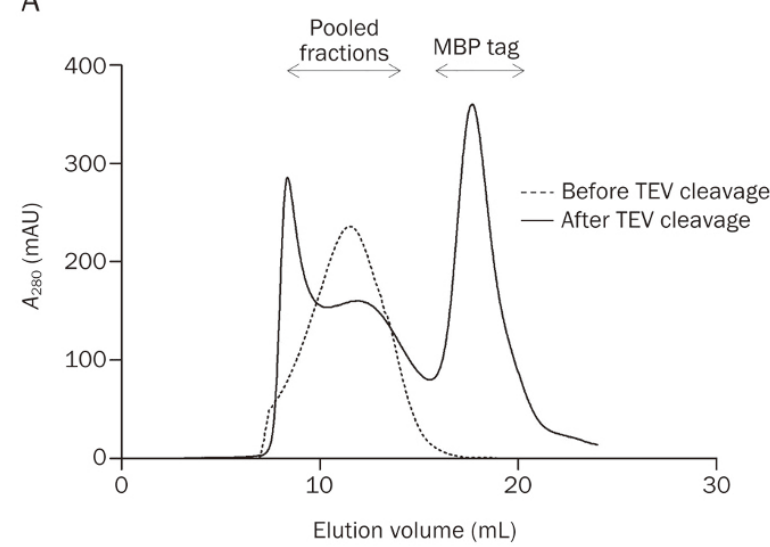

B

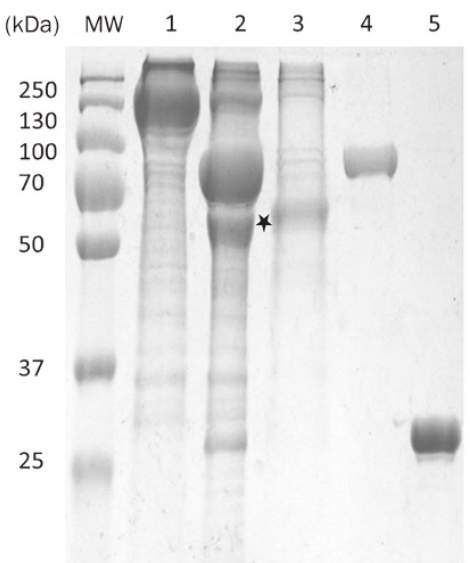

Figure 5. Large-scale expression and purification of the WT $\alpha 7-n A C h R$. (A) Size-column purification of the MBP-tagged (before TEV cleavage) Taeniopygia guttata and untagged (after TEV cleavage) $\alpha 7$-nAChR. (B) SDS-PAGE analysis of the elution fractions from the amylose column (lane 1), the sample digested with TEV protease (lane 2), the pooled fractions from size-exclusion chromatography of the TEV-digested sample (lane 3), the doubleMBP tag (lane 4), and TEV protease (lane 5).

A

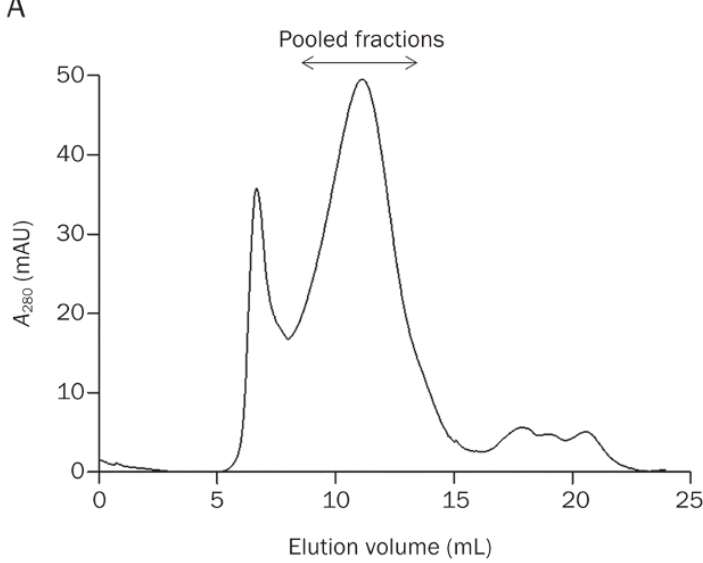

C

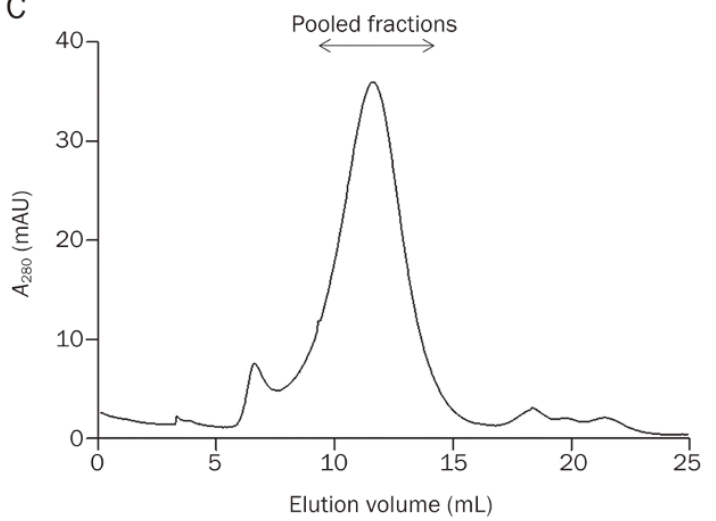

B (kDa) MW

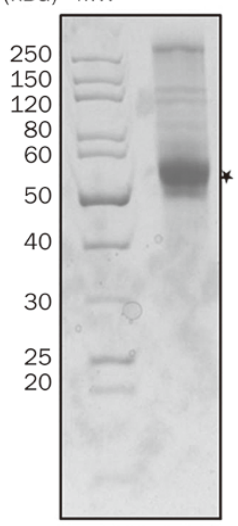

D (kDa) MW

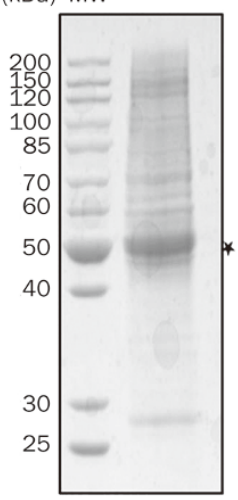

Figure 6. Analytical size-exclusion chromatography (SEC) and SDS-PAGE analysis of the optimized $\alpha 7-n A C h R$. The Taeniopygia guttata glycosylation

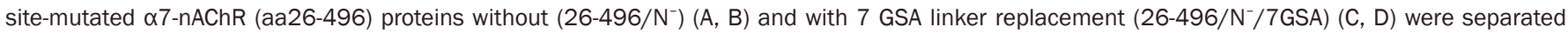
on a Superose $610 / 300 \mathrm{GL}$ gel filtration column (GE Healthcare) at a flow rate of $0.5 \mathrm{~mL} / \mathrm{min}$. $\alpha 7$-nAChRs from the pooled fractions after SEC were detected using SDS-PAGE (B, D). 
dye in the native state, but these residues become exposed upon thermal denaturation of the protein. The wild-type (WT) receptor and the C-terminally truncated receptors displayed similar melting temperatures (Tm) between $59-60^{\circ} \mathrm{C}$ (Figure 7A). The glycosylation site-mutated receptors and the receptor in which the intracellular loop between TM3 and TM4 was replaced with a $7 \times$ GSA linker displayed only a slightly decreased $\mathrm{Tm}$ of $58^{\circ} \mathrm{C}$ (Figure 7A). Notably, inclusion of the antagonists a-bungarotoxin and methyllycaconitine increased thermal stability by $4-5^{\circ} \mathrm{C}$, whereas the agonist epibatidine greatly decreased the thermal stability of the receptor (Figure $7 \mathrm{~B})$. The $15^{\circ} \mathrm{C}$ difference in Tm between antagonist- and agonist-bound receptors indicates that the antagonist-bound functions and the rational design of subtype-specific drugs. However, attempts to solve the crystal structures of these membrane proteins faced many technical challenges. One of the major obstacles was the preparation of $\mathrm{nAChR}$ proteins in sufficient quantity and quality. This study reported our extensive efforts in the expression and purification of the a7-nAChR and the biochemical and EM characterization of the purified receptor.

Several attempts were made to express full-length pLGICs from different species in prokaryotic and eukaryotic expression systems. ELIC ${ }^{[14]}$ and GLIC ${ }^{[15]}$ were expressed in bacteria as fusion proteins with maltose-binding protein, which yielded water-soluble ligand-binding proteins with sizes correspond-

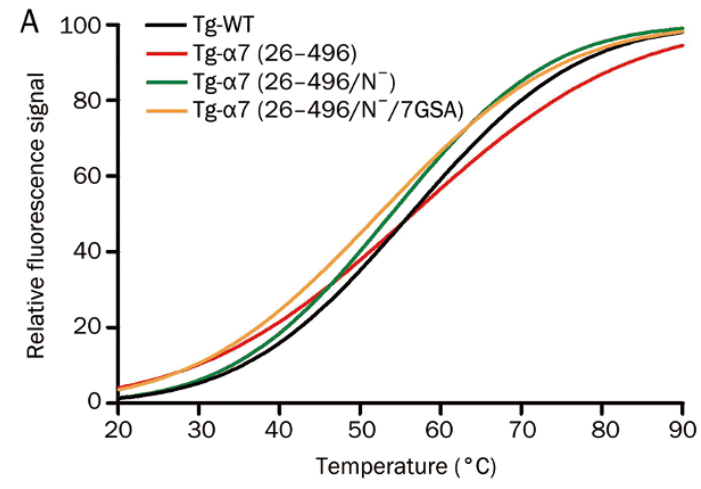

\begin{tabular}{lc}
\multicolumn{1}{c}{ Construct } & $\operatorname{Tm}\left({ }^{\circ} \mathrm{C}\right)$ \\
\hline Tg-WT & 60 \\
Tg- $\alpha 7(26-496)$ & 59 \\
Tg- $\alpha 7\left(26-496 / N^{-}\right)$ & 58 \\
Tg- $\alpha 7\left(26-496 / \mathrm{N}^{-} / 7 \mathrm{GSA}\right)$ & 58
\end{tabular}

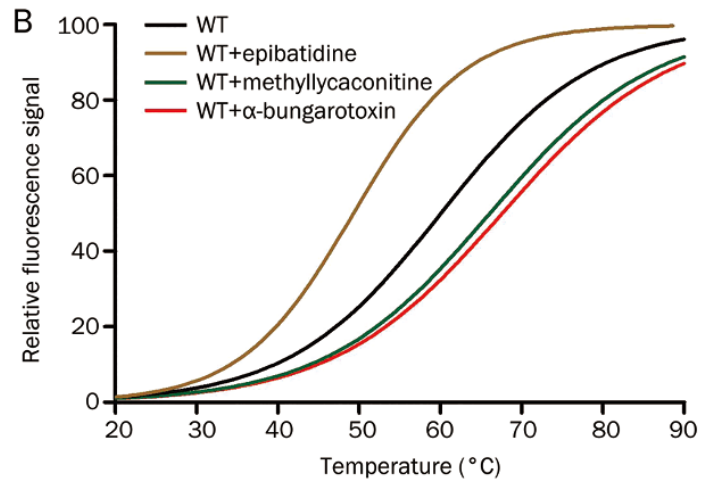

\begin{tabular}{lccc}
\multicolumn{1}{c}{ Ligand } & $\begin{array}{c}\text { Biological } \\
\text { activity }\end{array}$ & $K_{\mathrm{i}}(\mathrm{nmol} / \mathrm{L})$ & $\mathrm{Tm}\left({ }^{\circ} \mathrm{C}\right)$ \\
\hline WT & - & - & 60 \\
Epibatidine & Agonist & $16-22$ & 50 \\
Methyllycaconitine & Antagonist & $7.3-13.3$ & 64 \\
$\alpha$-Bungarotoxin & Antagonist & $0.48-1.06$ & 65
\end{tabular}

Figure 7. Thermal stability profiles of the purified $\alpha 7-n A C h R$. (A) Proteins from various optimized constructs; (B) Effect of different ligands on the thermal stability of purified $\alpha 7$-nAChRs.

conformation (presumably the closed channel conformation) is more stable than the agonist-bound (presumably open channel) conformation. This result is consistent with the fact that the $\mathrm{AAChR}$ channel is only transiently open upon the binding of the physiological agonist acetylcholine.

We also performed negative-stain EM analysis of the purified receptor that had a $7 \times$ GSA linker between TM3 and TM4. The EM images revealed a wide distribution of aggregating states of the receptor complex with a fraction of single complexes, which displayed a ring-like structure with a central hole (Figure 8). The ring-like structure observed in EM analysis resembled the pentameric structure of the nAChR ECD structure, which is consistent with the assembly of the nAChR channel.

\section{Discussion}

The nicotinic acetylcholine receptors are the best-studied members in the pLGIC superfamily. Detailed knowledge of their structures would facilitate the understanding of their

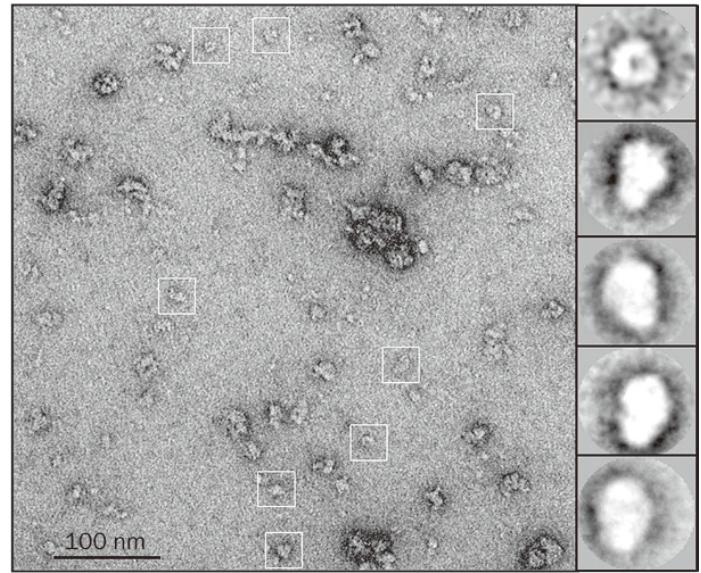

Figure 8. Representative negative-stain EM images of purified $\alpha 7$-nAChRs (26-496/ $\left.\mathrm{N}^{-} / 7 \mathrm{GSA}\right)$. Here, $\alpha 7-n A C h R$ particles are indicated by white boxes, and typical class averages of top and side views are shown in the right panel. The top view image shows a ring-like structure feature with a central hole. 
ing to pentamers. Breakthrough studies were also made for GluCl ${ }^{[16]}$ and $5-\mathrm{HT}_{3 \mathrm{~A}}{ }^{[18]}$, which were expressed in insect cells and HEK293s cells, respectively. Our work demonstrated that a7-nAChRs can be expressed in HEK293F cells using a BacMam expression system and receptor modifications, including C-terminal truncation, replacement of the intracellular loop with GSA linkers, and the removal of glycosylation sites, which improved protein expression and behavior. The purified protein appeared to be quite stable with a Tm approaching $60^{\circ} \mathrm{C}$. The presence of antagonists further increased the thermal stability of the purified receptor, but the presence of agonist decreased the stability, which implies that the openchannel conformation is less stable than the closed conformation. EM analysis of single isolated complexes revealed a ringlike structure that is consistent with the pentameric assembly of the channel.

Our primary goal was to obtain a crystal structure of a7-nAChR. However, we failed to generate crystals of this important protein complex despite our exhaustive efforts in crystallization screening. EM images (Figure 8) indicated that the purified receptor from the final gel filtration fractions contained many forms of micro-aggregation, which may prevent crystallization. The purified receptor appeared to have high thermal stability and respond to the presence of agonist and antagonist, but it was still prone to aggregation that prevented crystallization. There are several ways to overcome this aggregation problem, including protein engineering, detergent and additive screening, and the inclusion of antibody fragments into crystallization. The recent success of nanobodyfacilitated crystallization of the $5-\mathrm{HT}_{3 \mathrm{~A}}$ complex is particularly encouraging, and this technique could be similarly applied to a7-nAChRs. The expression and purification methods reported here should pave the way for future high-resolution studies of a7-nAChR by crystallization or single-particle cryoEM analysis.

\section{Acknowledgements}

This work was supported in part by the Jay and Betty Van Andel Foundation, Ministry of Science and Technology (China) grants 2012ZX09301001 and 2012CB910403, 2013CB910600, XDB08020303, 2013ZX09507001, XDB08030201, 13JC1406300, 31222016, 31270771, and Shanghai Science and Technology Committee grant 13ZR1447600, 13JC1406300. We thank Dr Yi SHI for advice on figure preparation and Yueming ZHENG and Zhao-bing GAO for consultation on experimental design.

\section{Author contribution}

H Eric XU and Hao CHENG designed research; Hao CHENG, Si-wei ZHANG and Zhong-shan WU performed research; Yao CONG, Chen FAN and Zhi-cheng CUI contributed electron microscopy analysis; Cheng-hai ZHANG and Yi JIANG contributed BacMam expression system; H Eric XU, Yao CONG and Hao CHENG analyzed data; H Eric XU, Karsten MELCHER and Hao CHENG wrote the paper.

\section{References}

1 Hurst R, Rollema H, Bertrand D. Nicotinic acetylcholine receptors: from basic science to therapeutics. Pharmacol Ther 2013; 137: 22-54.

2 Miller PS, Smart TG. Binding, activation and modulation of Cys-loop receptors. Trends Pharmacol Sci 2010; 31: 161-74.

3 Fasoli F, Gotti C. Structure of neuronal nicotinic receptors. Curr Top Behav Neurosci 2015; 23: 1-17.

4 Paterson D, Nordberg A. Neuronal nicotinic receptors in the human brain. Prog Neurobiol 2000; 61: 75-111.

5 Role LW, Berg DK. Nicotinic receptors in the development and modulation of CNS synapses. Neuron 1996; 16: 1077-85.

6 Mehta M, Adem A, Kahlon MS, Sabbagh MN. The nicotinic acetylcholine receptor: smoking and Alzheimer's disease revisited. Front Biosci (Elite Ed) 2012; 4: 169-80.

7 Corringer PJ, Le Novere N, Changeux JP. Nicotinic receptors at the amino acid level. Annu Rev Pharmacol Toxicol 2000; 40: 431-58.

8 Gotti C, Clementi F. Neuronal nicotinic receptors: from structure to pathology. Prog Neurobiol 2004; 74: 363-96.

9 Hogg RC, Raggenbass M, Bertrand D. Nicotinic acetylcholine receptors: from structure to brain function. Rev Physiol Biochem Pharmacol 2003; 147: 1-46.

10 Wallace TL, Bertrand D. Alpha7 neuronal nicotinic receptors as a drug target in schizophrenia. Expert Opin Ther Targets 2013; 17: 139-55.

11 Yakel JL. Gating of nicotinic ACh receptors: latest insights into ligand binding and function. J Physiol 2010; 588: 597-602.

12 Li SX, Huang S, Bren N, Noridomi K, Dellisanti CD, Sine SM, et al. Ligand-binding domain of an alpha7-nicotinic receptor chimera and its complex with agonist. Nat Neurosci 2011; 14: 1253-9.

13 Unwin N. Refined structure of the nicotinic acetylcholine receptor at 4 A resolution. J Mol Biol 2005; 346: 967-89.

14 Hilf RJ, Dutzler R. X-ray structure of a prokaryotic pentameric ligandgated ion channel. Nature 2008; 452: 375-9.

15 Hilf RJ, Dutzler R. Structure of a potentially open state of a protonactivated pentameric ligand-gated ion channel. Nature 2009; 457: 115-8.

16 Hibbs RE, Gouaux E. Principles of activation and permeation in an anion-selective Cys-loop receptor. Nature 2011; 474: 54-60.

17 Miller PS, Aricescu AR. Crystal structure of a human GABAA receptor. Nature 2014; 512: 270-5.

18 Hassaine G, Deluz C, Grasso L, Wyss R, Tol MB, Hovius R, et al. X-ray structure of the mouse serotonin $5-\mathrm{HT}_{3}$ receptor. Nature 2014; 512: 276-81.

19 Picciotto MR, Higley MJ, Mineur YS. Acetylcholine as a neuromodulator: cholinergic signaling shapes nervous system function and behavior. Neuron 2012; 76: 116-29.

20 Changeux JP. Nicotine addiction and nicotinic receptors: lessons from genetically modified mice. Nat Rev Neurosci 2010; 11: 389-401.

21 Rahman S. Nicotinic receptors as therapeutic targets for drug addictive disorders. CNS Neurol Disord Drug Targets 2013; 12: 633-40.

22 Thompson JD, Higgins DG, Gibson TJ. CLUSTAL W: improving the sensitivity of progressive multiple sequence alignment through sequence weighting, position-specific gap penalties and weight matrix choice. Nucleic Acids Res 1994; 22: 4673-80.

23 Alexandrov Al, Mileni M, Chien EY, Hanson MA, Stevens RC. Microscale fluorescent thermal stability assay for membrane proteins. Structure 2008; 16: 351-9.

24 Tang G, Peng L, Baldwin PR, Mann DS, Jiang W, Rees I, et al. EMAN2: an extensible image processing suite for electron microscopy. J Struct Biol 2007; 157: 38-46.

25 Ludtke SJ, Baldwin PR, Chiu W. EMAN: semiautomated software for 
high-resolution single-particle reconstructions. J Struct Biol 1999; 128: 82-97.

26 Chaudhary S, Pak JE, Pedersen BP, Bang $\sqcup$, Zhang LB, Ngaw SM, et al. Efficient expression screening of human membrane proteins in transiently transfected human embryonic kidney 2935 cells. Methods 2011; 55: 273-80.

27 Liao M, Cao E, Julius D, Cheng Y. Structure of the TRPV1 ion channel determined by electron cryo-microscopy. Nature 2013; 504: 107-12.

28 Hassaine G, Deluz C, Tol MB, Li XD, Graff A, Vogel H, et al. Large scale expression and purification of the mouse 5-HT3 receptor. Biochim Biophys Acta 2013; 1828: 2544-52.

29 Dukkipati A, Park HH, Waghray D, Fischer S, Garcia KC. BacMam system for high-level expression of recombinant soluble and membrane glycoproteins for structural studies. Protein Expr Purif 2008; 62: $160-70$.

30 Jansen M, Bali M, Akabas MH. Modular design of Cys-loop ligandgated ion channels: functional 5-HT3 and GABA rho1 receptors lacking the large cytoplasmic M3M4 loop. J Gen Physiol 2008; 131: 137-46. 